\title{
Flipping and spinning: Spatial transformation procedures in the identification of rotated natural objects
}

\author{
JANICE E. MURRAY \\ University of Otago, Dunedin, New Zealand
}

\begin{abstract}
The proposal that identification of inverted objects is accomplished by either a relatively slow rotation in the picture plane or a faster rotation in the depth plane about the horizontal axis was tested. In Experiment 1, subjects decided whether objects at $0^{\circ}$ or $180^{\circ}$ corresponded to previously learned normal views of the upright objects, or were mirror images. Instructions to mentally flip an inverted object in the depth plane to the upright produced faster decision times than did instructions to mentally spin the object in the picture plane. In Experiment 2, the effects of orientation were compared across an object-naming task and a normal-mirror task for six orientations from $0^{\circ}$ to $300^{\circ}$. In the normal-mirror task, objects at $180^{\circ}$ were cued for rotation in the picture plane or in the depth plane in equal numbers. The naming function for one group of subjects did not differ from the normalmirror function where inverted objects had been mentally rotated to the upright. For both functions, response time (RT) increased linearly from $0^{\circ}$ to $180^{\circ}$ and the slopes did not differ. The naming function for a second group of subjects did not differ from the normal-mirror function where inverted objects had been mentally flipped to the upright. For both functions, RT increased linearly at a similar rate from $0^{\circ}$ to $120^{\circ}$, but decreased from $120^{\circ}$ to $180^{\circ}$. The results are discussed in terms of theories of orientation-specific identification.
\end{abstract}

Much of the contemporary research investigating visual object recognition has sought to determine the representations and processes used to identify objects. One approach has been to study the effects of picture-plane rotation on identification of line drawings of objects. Typically, objects are shown at $0^{\circ}, 60^{\circ}, 120^{\circ}, 180^{\circ}, 240^{\circ}$, and $300^{\circ}$ of clockwise rotation, and subjects are asked to name the individually presented objects as quickly as possible. The resulting naming times indicate that the initial identification of rotated objects is highly sensitive to the degree of rotation of the object from the canonical view. When line drawings of objects are displayed for the first time in an experiment, naming time increases monotonically as a function of the angular departure of the rotated object from the upright (see, e.g., Jolicoeur, 1985; Maki, 1986; Murray, 1995b; Murray, Jolicoeur, McMullen, \& Ingleton, 1993). Notably, this increase in naming time is linear for orientations between $0^{\circ}$ and $120^{\circ}$. However, the naming times observed for $180^{\circ}$ rotations do not conform to the pattern predicted by linear extrapolation of the results from the other orientations. Sometimes naming times at $180^{\circ}$ show only a slight increase relative

This research was supported by grants from the Natural Sciences and Engineering Research Council Canada, and the University of Otago Research Committee. I gratefully acknowledge the assistance of P. Hanning and C. Harrison with data collection. I thank J. Hamm, R. Klein, P. McMullen, and J. Miller for helpful discussions, and G. Loftus, G. Snodgrass, and an anonymous reviewer for comments on an earlier draft of the manuscript. Correspondence should be addressed to J. Murray, Department of Psychology, University of Otago, Box 56. Dunedin, New Zealand (e-mail: jmur@rivendell.otago.ac.nz). to naming times at $120^{\circ}$ of clockwise or counterclockwise rotation (see, e.g., Jolicoeur \& Milliken, 1989; Murray, 1995b), and often are much faster, producing an overall M-shaped naming function (see, e.g., Jolicoeur, 1985; Murray, 1995a, 1995b).

The pattern of results for orientations between $0^{\circ}$ and $120^{\circ}$ has been interpreted as evidence in favor of orientation-specific accounts of object recognition (Jolicoeur, 1990). It has been suggested that for many objects, there is a typical viewing orientation, most often upright, and that for each object a single representation is stored at this canonical orientation. These long-term memory representations are ones in which the spatial relations among parts are stored in terms of upright internal axes. Successful object recognition requires that the spatial relations of the parts in the input representation be mapped onto those of the stored representation. When the orientation of the input representation departs from that of the stored representation, normalization operations must be enacted to correct for that departure. Transformation of the input representation of a rotated object to the upright is required to allow for comparison of the input representation with orientation-dependent representations in memory (e.g., Jolicoeur, 1985; Tarr \& Pinker, 1989; Ullman, 1989).

The fact that naming time increases linearly with orientational disparity from $0^{\circ}$ to $120^{\circ}$ has led to the suggestion that "mental rotation" is the process that aligns the input representation with the stored representations (Jolicoeur, 1985). Mental rotation is considered to be an ana$\log$ transformation of an image and is characterized as a continuous rotation of a nonupright image to the upright 
(Shepard \& Cooper, 1982). Typically, mental rotation has been studied in tasks requiring some decision about the handedness of the stimulus, such as whether it is normal or mirror image (for a review, see Shepard \& Cooper, 1982). In these tasks, response time (RT) increases linearly and the symmetric function peaks at $180^{\circ}$. Jolicoeur $(1985,1988)$ obtained the characteristic mental rotation function in a task requiring a decision regarding whether an object in profile faced left or right. Comparing the magnitude of the orientation effect for the leftright task with that for an object-naming task, he found that the slopes of the functions were similar across the two tasks between $0^{\circ}$ and $120^{\circ}$. This similarity was taken as evidence supporting the notion that mental rotation is the mechanism underlying the orientation effect observed in object identification tasks, at least within the $0^{\circ}-120^{\circ}$ range. Clear differences were observed across the two tasks, however, when RTs for objects at $180^{\circ}$ were considered. In the left-right task, RT increased monotonically across the full orientation range, peaking at $180^{\circ}$. In contrast, whereas RT increased linearly in the naming task from $0^{\circ}$ to $120^{\circ}$, it decreased from $120^{\circ}$ to $180^{\circ}$.

Jolicoeur (1990) has argued that the faster naming times at $180^{\circ}$ reflect the operation of a second set of identification processes that operate in parallel with the normalization processes necessary for successful identification of objects between $0^{\circ}$ and $120^{\circ}$ described above. This second set of processes makes use of representations in which orientation-free features and parts serve as the basis for object identification, and spatial relations among the features and parts are not encoded. For objects at $180^{\circ}$, the top-bottom axis is coincident with that of the upright canonical representation. Jolicoeur has suggested that this axis alignment may provide the opportunity for some successful feature matches independent of orientation, and that these orientation-invariant feature extraction processes result in the faster naming times for objects at $180^{\circ}$.

In the present study, a second possible explanation of the faster naming times for inverted objects is investigated: Like naming of objects at orientations between $0^{\circ}$ and $120^{\circ}$, naming of objects at $180^{\circ}$ is also accomplished by transformation of an image of the rotated object to the upright for comparison with orientation-specific representations in memory. What differs is the transformation process itself. In the case of objects at orientations other than $180^{\circ}$, the transformation process appears to be one of rotation in the picture plane. At $180^{\circ}$, the identity of the object could be established through a reflection transformation. Rather than rotation in the picture plane, rotation could occur in the depth plane about the horizontal axis of the inverted object. This type of transformation can be termed mental flipping (see Koriat, Norman, \& Kimchi, 1991). Although some features may be successfully matched because of the alignment of the inverted object and canonical representation top-bottom axes, many features of the inverted object will not directly match those of the upright representation. The combination of axis alignment and feature mismatch may be sufficient to signal a reflection transformation to establish object iden- tity at $180^{\circ}$. Reflection transformations may be restricted to inverted objects since the alignment of top-bottom axes is unique to objects at $0^{\circ}$ and $180^{\circ}$. For objects at other orientations, reflection transformations are possible but would need to be followed by a rotation in the picture plane in order for the object to be compared with the canonical representation. A single rotation in the picture plane would seem to be the optimal rotation strategy for identification of objects at orientations other than $180^{\circ}$.

Support for the notion of a rotation-in-depth processing strategy for transformation of inverted objects can be found in a number of studies. Bressan and Vicario (1984) reported that subjects may find rotations in the depth plane about the horizontal and vertical axes to be more natural transformations than rotation in the picture plane. In their task, subjects were asked to demonstrate the sequence of operations that had been performed to produce the rotated letters they viewed. To produce an inverted letter, subjects showed a preference for two reflections, one about the horizontal axis and one about the vertical axis, over a single rotation in the picture plane.

Evidence suggests that depth rotation about the horizontal axis may be a faster process as well. In a visualization experiment, Shepard and Feng instructed subjects to report the outcome of a specified spatial transformation of auditorily presented letters (Shepard, 1975). The instruction to perform a reflection about either the horizontal or vertical axis reliably produced a faster visualization time than did the instruction to rotate the letter $180^{\circ}$ in the picture plane. In their well-known experiments, Shepard and Metzler (1971) examined the effects of rotation on 3-dimensional arm-like figures formed from small cubes. On any trial, subjects were asked to decide if the two presented figures were the same shape, ignoring any differences in orientation. Generally there was no difference in the functions for pairs that differed by rotation in the picture plane or by rotation in the depth plane, but the slope of the linear function relating angle of rotation and RT did depend on the plane of rotation when the rotation occurred about a "natural" axis for depth-rotated pairs. If the axis of rotation was about the central and longest row of cubes constituting the figure, the slope of the RT function for depth plane rotation was shallower than that for picture plane rotation (Metzler \& Shepard, 1974). Additional work by Parsons (1987) also indicates a faster mental flipping process. Using the Shepard and Metzler (1971) figures, Parsons explored differences in orientation between stimulus pairs about 13 different axes or planes. He found that for all axes, the time to discriminate mirror-reflected and identical pairs of figures varied as a function of the difference in orientation, but the slope of the function for rotations about the horizontal axis was considerably shallower than the slopes for rotations about any other axis, including rotation in the picture plane. Together, these results (1) suggest that mental flipping is a transformation strategy available to subjects and (2) are consistent with the proposition that alignment through rotation in depth is responsible for the faster naming times for objects at $180^{\circ}$. 
As noted, in contrast to naming functions, the RT function in mental rotation tasks requiring a handedness judgment peaks at $180^{\circ}$, as would be predicted by rotation in the picture plane. This difference between handedness and naming tasks can be accounted for by considering the consequences of the two types of rotation and task demands. Consider an inverted tea cup with its handle on the right side. If the cup is rotated to the upright in the depth plane, the handle remains on the right side of the upright cup. If, however, it is rotated in the picture plane, the handle is now on the left side of the upright cup. In mental rotation tasks, it is assumed that rotation is in the picture plane for all orientations and that trial-by-trial feedback provided to the subject concerning accuracy of response is based on that assumption. If subjects initially adopt the strategy of rotating in the depth plane, their resulting images would yield the opposite answer to a picture plane rotation as described above. In mental rotation tasks, it is likely that subjects learn that the correct response is produced by a rotation in the picture plane and adopt such a rotation process. In effect, choice of rotation route is constrained by the task involving left-right discriminations. No such constraint is imposed in naming tasks if object identification is insensitive to the handedness of an object. The naming task permits the potential use of either rotation route to identification of objects at $180^{\circ}$.

The available data from identification tasks provide some indication that both routes may be used, with some subjects rotating inverted objects in the depth plane and others in the picture plane. As described, in many experiments, inverted objects are named much faster than are objects rotated $120^{\circ}$ from the upright. In other experiments, naming times for objects at $180^{\circ}$ show a modest decrease or a small increase relative to objects at $120^{\circ}$ and $240^{\circ}$. This variation across experiments would be expected if, in general, most individuals adopt a mental flipping strategy but some individuals choose to rotate in the picture plane. That the variation is subject based rather than stimulus based is suggested by the observation that these different patterns can be observed when the stimulus set is the same and the subject sample differs (e.g., Murray, 1995a).

The nature of the response in the identification task precludes any exact determination of the path of rotation for objects at $180^{\circ}$. In an identification task, individuals simply name the inverted objects, and this naming response reveals nothing about whether the object was rotated in the picture plane or in the depth plane. The path of rotation, however, can be determined in a left-right discrimination task. Rotations in the picture and depth planes produce different left-right views, as noted, and consequently a subject's left-right discrimination response provides an indication of rotation route. This suggests that a comparison of left-right tasks (where path of rotation can be determined) with identification tasks can be used to investigate the nature of the processes involved in the identification of rotated objects at all orientations, but particularly those at $180^{\circ}$. The approach of comparing the magnitude of orientation effects across tasks has been used by Jolicoeur $(1985,1988)$ to explore the possibility that the linear portion of the naming function between $0^{\circ}$ and $120^{\circ}$ reflects a process of mental rotation that is common to both naming and left-right discrimination tasks. In the research reported herein, performance on a naming task was compared with performance on a normal-mirror discrimination task where subjects were instructed to rotate inverted objects in the picture plane or in the depth plane about the horizontal axis. If the overall shape and slope of the orientation function for naming is found to be similar to that for the left-right task under one or the other rotation instructions, this would serve to identify the rotation route used in the identification of inverted objects. Additionally, it would suggest that the processes underlying identification of rotated objects are the same as those underlying left-right tasks.

\section{EXPERIMENT 1}

In Experiment 1, it was first established whether rotation in the depth plane about the horizontal axis is a faster process than is rotation in the picture plane for a normal-mirror discrimination task involving natural objects. Subjects learned the "normal" view assigned each of six objects and then made normal-mirror judgments about the objects presented at $0^{\circ}$ and $180^{\circ}$. For objects presented at $180^{\circ}$, subjects were instructed to either mentally flip the inverted object to the upright or rotate it in the picture plane in order to make the normal-mirror decision.

\section{Method}

Subjects. Fifteen students ( 7 females) of Carleton University participated as subjects. All were native speakers of English without previous experience with the stimuli, and had normal or correctedto-normal vision. Each subject was tested in a single session of approximately $30 \mathrm{~min}$

Stimuli and Apparatus. The stimuli were six line drawings chosen from Snodgrass and Vanderwart (1980). The depicted objects were a teacup, a football helmet, a kettle, a watering can, a harp, and a spinning wheel, with each object characterized by a distinct feature on either the left or right side. In the "normal" view of each object, the asymmetrical feature was on the left side for three objects and on the right side for the other three objects, as illustrated in Figure 1. Stored images were created by scanning each drawing; they were then edited to produce uniform images for $0^{\circ}$ and $180^{\circ}$ of clockwise rotation. In addition, mirror-image versions of the drawings were produced for each of the orientations. The drawings were presented on a Zenith 1492 VGA color monitor as black line drawings on a light gray background, and subtended an average of $3^{\circ}$ of visual angle. Stimulus presentation and collection of responses were controlled by an NEC Powermate 386 computer using Micro Experimental Laboratory software (Schneider, 1988).

Procedure. Subjects were first taught the normal versions of the six objects. Upright drawings were individually displayed on the computer screen for $4 \mathrm{sec}$ each, and the sequence of six presentations was shown three times. Subjects were informed that the presented view represented the normal version of each object and that later they would be asked to distinguish between normal and mirror-image versions. Following this training, subjects were pro- 

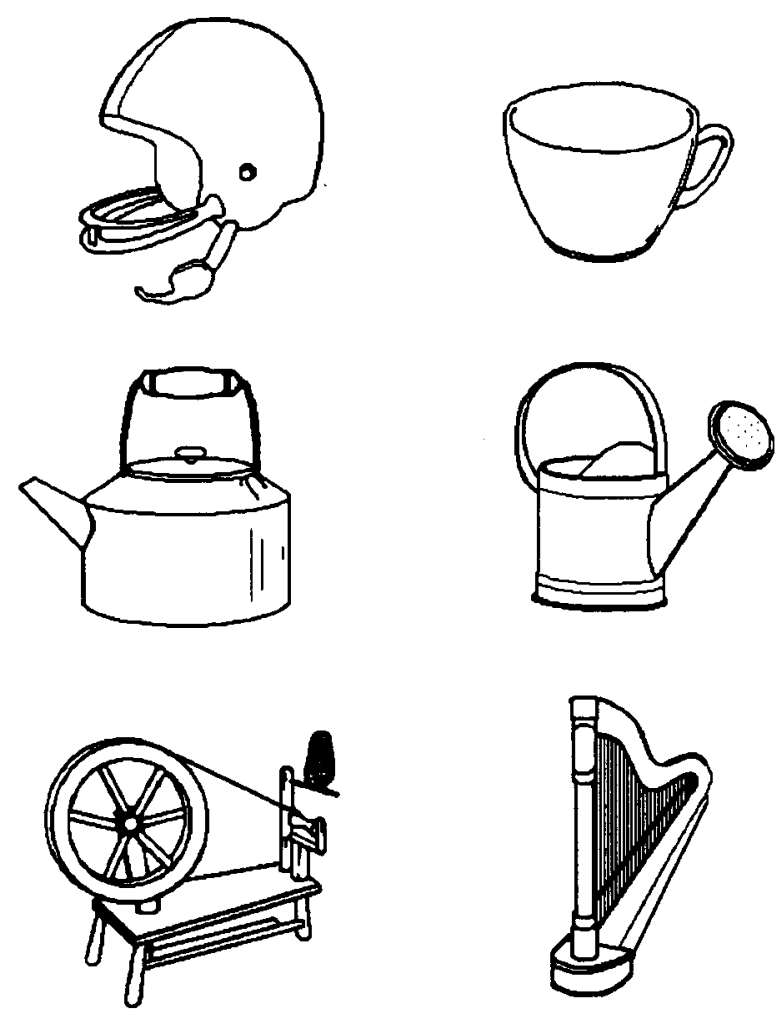

Figure 1. Normal upright versions of the stimuli used in the normal-mirror task of Experiment 1. In Experiment 2, the football helmet was replaced by a frying pan.

vided with the opportunity to study printed copies of the objects until they were satisfied that they had learned the normal view.

The subjects were next tested on normal and mirror-image versions of each upright object and asked to make a normal-mirror judgment as quickly as possible. Each object was presented three times in each view for a total of 36 randomly presented trials. At the beginning of a trial, the subject viewed a fixation cross in the center of the screen. A keypress by the subject resulted in a blank screen for $213 \mathrm{msec}$, during which time the stimulus was written to the screen. The screen was then turned on at the top of the refresh cycle to reveal the drawing, which remained in view until the subject responded by pressing the "N" key (normal) or "B" key (mirror image) on the keyboard. Response latency was measured to the nearest millisecond from the onset of the drawing to the subject's response. In order to proceed to the next part of the experiment, subjects were required to attain a mean RT of less than $1 \mathrm{sec}$ with fewer than two errors. If a subject failed to meet these criteria, he or she was asked to study the normal views again and was then retested. Two subjects were unable to meet the criteria after several attempts and were replaced.

Following successful completion of upright training, subjects were introduced to inverted versions of the objects. They were informed that there were two ways in which an object could be rotated to the upright position. Rotation out of the picture plane was described as a "flip," and rotation in the picture plane was described as a "spin." Subjects were then shown that flip and spin rotations produced different normal/mirror-image versions. Examples of each type of transformation were demonstrated by the experimenter with a drawing of one object on an overhead transparency before inverted and upright versions of each object were presented twice on the computer screen. One presentation showed the upright version resulting from a flip, and the other revealed the upright version for the same inverted object after a spin. Testing of depth and picture plane rotation followed. Each trial was initiated by a subject keypress. A cue indicating type of transformation required for the ensuing inverted drawing replaced the fixation cross. The cue-flip or spin-was displayed for $1,500 \mathrm{msec}$. After a 213-msec blank interval, an inverted object was presented and remained in view until the subject responded by pressing the " $N$ " or " $B$ " key on the keyboard. Subjects were instructed to decide whether the inverted object would be normal ("N" key) or mirror image ("B" key) if it were viewed upright following the cued transformation, and were reminded that the two transformations, flip and spin, produced different versions and hence, different responses. Accuracy and RT feedback were provided on each trial. Twelve trials were randomly presented in all, six requiring picture plane spins and six requiring depth plane flips. Half of the trials for each type of transformation required normal responses and half required mirror image. If subjects made more than one error, the 12 trials were repeated.

Finally, subjects participated in the experimental trials, where they made normal-mirror decisions for both upright and inverted objects. In a block of 36 trials, each object was presented equally often upright, inverted flip, and inverted spin. Half the trials of each type required normal responses and half required mirrorimage responses. Trials proceeded as described above with the addition that "xxxx" cued an upright trial. Subjects received two blocks for a total of 72 trials.

\section{Results and Discussion}

RTs of less than 300 and greater than $5,000 \mathrm{msec}$ were considered outliers. Application of this criterion resulted in the rejection of less than $1 \%$ of the data. Mean RTs for correct responses were calculated for each subject in each transformation and version condition. The mean RTs and error rates are presented in Table $1 .^{1}$

As can be seen in Table 1, transformation (upright, inverted flip, inverted spin) had a significant effect on RT. Subjects took $512 \pm 62 \mathrm{msec}$ longer on average to make the normal-mirror judgment in the inverted-spin condition than in the upright condition. Responses in the flip condition were also slower than those in the upright condition, by $289 \pm 45 \mathrm{msec}$. Most importantly, the time to make a normal-mirror response under flip instructions was $223 \pm 45 \mathrm{msec}$ faster than the time to make the judgment under spin instructions.

In general, subjects responded to normal views more rapidly than to mirror-image views, as is typically found in mental rotation tasks. This difference between normal and mirror image was much greater in the spin condition $(126 \pm 31 \mathrm{msec})$ than in either the upright $(59 \pm 22 \mathrm{msec})$ or the flip conditions $(23 \pm 44 \mathrm{msec})$. This pattern of ef-

Table 1

Mean Response Time \pm Standard Error (in Milliseconds; $\mathrm{RT} \pm S E$ ) and Mean Percent Error (MPE) for Normal and Mirror-Image Objects in Each Transformation Condition of Experiment 1

\begin{tabular}{|c|c|c|c|c|c|c|}
\hline \multirow[b]{2}{*}{ Task } & \multicolumn{2}{|c|}{$0^{\circ}$} & \multicolumn{2}{|c|}{$\begin{array}{c}\text { Inverted } \\
\text { Flip } \\
\end{array}$} & \multicolumn{2}{|c|}{$\begin{array}{c}\text { Inverted } \\
\text { Spin }\end{array}$} \\
\hline & $\mathrm{RT} \pm S E$ & MPE & $\mathrm{RT} \pm S E$ & MPE & $\mathrm{RT} \pm S E$ & MPE \\
\hline Normal & $886 \pm 26$ & 6.7 & $1,193 \pm 73$ & 5.0 & $1,365 \pm 71$ & 6.1 \\
\hline Mirror image & $945 \pm 30$ & 3.9 & $1,216 \pm 66$ & 5.6 & $1,491 \pm 78$ & 8.3 \\
\hline$M$ & $916 \pm 26$ & 5.0 & $1,205 \pm 66$ & 5.3 & $1,428 \pm 73$ & 7.3 \\
\hline
\end{tabular}


fects was not replicated in Experiment 2. A similar analysis of errors revealed no significant results.

The results of Experiment 1 are clear. First, the time to make a normal-mirror judgment for an inverted object is slower than that for an upright object. This is true regardless of whether the instruction to rotate the object to the upright is for rotation in the picture plane or in the depth plane. Second, the time taken to rotate an inverted object to the upright is considerably less under instructions to mentally flip. These findings are consistent with previous research investigating normal-mirror discriminations in simultaneously presented pairs of abstract 3-dimensional shapes (Metzler \& Shepard, 1974; Parsons, 1987).

\section{EXPERIMENT 2}

The results of Experiment 1 indicate that an image of an inverted object can be rotated to the upright through a rotation in the depth plane for comparison with stored representations, and that this process is substantially faster than rotation of the same image to the upright in the picture plane. In Experiment 2, the possibility that relatively fast RTs for objects at $180^{\circ}$ in a naming task are also the result of similar rotation processes was explored.

Subjects first identified objects presented at orientations from $0^{\circ}$ to $300^{\circ}$ in $60^{\circ}$ steps under simple instructions to name the objects as quickly as possible. Following the naming task, subjects were tested in the normal-mirror task, as in Experiment 1, but using the full range of orientations from $0^{\circ}$ to $300^{\circ}$ in $60^{\circ}$ steps. For objects at $180^{\circ}$, rotation in both planes was again tested. Individual differences in the rotation route used to align an object at $180^{\circ}$ with a canonical representation provided a main focus for Experiment 2. As noted, an examination of identification RTs in past studies indicates considerable variability in naming performance at $180^{\circ}$ relative to performance at $120^{\circ}$. This variability may be accounted for if some individuals rotate $180^{\circ}$ objects in the picture plane, resulting in relatively slow RTs, while others rotate in the depth plane, resulting in relatively fast RTs. To assess this possibility, the RT data for naming at $180^{\circ}$ were used to establish two groups of subjects. One group consisted of individuals whose naming times at $180^{\circ}$ were slow relative to naming times at $120^{\circ}$ of clockwise or counterclockwise rotation. The second group comprised individuals who produced fast naming times at $180^{\circ}$ relative to naming times at $120^{\circ}$

The naming functions of subjects with relatively slow and relatively fast naming times at $180^{\circ}$ were then compared with the mental rotation functions derived from the normal-mirror task under instructions to mentally rotate and mentally flip objects at $180^{\circ}$. For any naming/ normal-mirror comparison, any observed similarity in the magnitude of the orientation effect would suggest that the process underlying the orientation effect in the normalmirror task also mediates the orientation effect in the naming task. If the fast naming times at $180^{\circ}$ are a consequence of a rotation-in-depth process, the overall shape and slope of the naming function for subjects with relatively fast identification times at $180^{\circ}$ should not differ from the mental rotation function derived from the normalmirror task under the instruction to mentally flip objects at $180^{\circ}$. This result would suggest a common underlying process of rotation in the picture plane for orientations between $0^{\circ}$ and $120^{\circ}$ (Jolicoeur, 1985, 1988), and a normalization process of depth rotation at $180^{\circ}$. Similarly, if slow naming times are the result of a rotation in the picture plane, the overall shape and slope of the naming function for subjects with relatively slow identification times at $180^{\circ}$ should not differ from the mental rotation function derived from the normal-mirror task under the instruction to mentally spin objects at $180^{\circ}$. This result would indicate that rotation in the picture plane was the process common to both tasks across the full orientation range.

\section{Method}

Subjects. Forty-two students of the University of Otago participated as subjects. All were native speakers of English and had normal or corrected-to-normal vision. None of the subjects had any previous experience with the stimuli. Testing for each subject took place in a session of approximately $50 \mathrm{~min}$.

Stimuli and Apparatus. The stimuli for the mirror-normal judgment were those used in Experiment 1 with the exception of the football helmet, which was replaced with a drawing of a frying pan (in the land of rugby, football helmets are not familiar objects). In addition to the normal and mirror-image views at $0^{\circ}$ and $180^{\circ}$, each drawing was also produced in its normal view at $60^{\circ}$, $120^{\circ}, 240^{\circ}$, and $300^{\circ}$ of clockwise rotation following the procedures outlined in Experiment 1. Drawings of 30 new objects were also created at $0^{\circ}, 60^{\circ}, 120^{\circ}, 180^{\circ}, 240^{\circ}$, and $300^{\circ}$ of clockwise rotation. These were combined with the normal views of the six objects of the mirror-normal task to provide a set of stimuli for a naming task. Accepted names for the naming stimuli are given in the Appendix. Drawings of an additional eight objects were used for practice trials. In addition to the apparatus in Experiment 1, a voice-activated relay connected to a microphone was used to detect naming latency.

Procedure. Subjects performed two tasks: naming and mirror normal. For the naming task, the 36 drawings of objects were each presented once in a single block of trials. There were equal numbers of objects at each of the six possible orientations from $0^{\circ}$ to $300^{\circ}$, and subjects received one 36-trial block. To control for any item-specific effects on orientation, six different naming blocks were created, with each object occurring once at each of the six orientations across blocks. Equal numbers of subjects were randomly assigned to each block. Thus, although a subject viewed an object at only one orientation in the naming task, across the entire group of subjects each object was presented equally often at each of the six orientations.

At the beginning of a naming trial, the subject viewed a central fixation cross on the screen. Following a subject-initiated keypress, the screen was blanked for a 213-msec interval, during which time the stimulus was drawn to the screen. At the end of this interval, the screen was turned on at the top of the refresh cycle to reveal the drawing, which remained in view until the subject responded. The object drawings were randomly presented. Subjects were instructed to name the displayed object as quickly and accurately as possible. Naming latency was measured from the onset of the display to the onset of the subject's verbal response. Responses were detected by the voice-activated relay and recorded by the com- 
Table 2

\begin{tabular}{|c|c|c|c|c|c|c|}
\hline \multicolumn{7}{|c|}{$\begin{array}{c}\text { Mean Response Time } \pm \text { Standard Error (in Milliseconds; } R T \pm S E \text { ) and } \\
\text { Mean Percent Error (MPE) for Normal and Mirror-Image Objects } \\
\text { in Each Transformation Condition of Experiment } 2\end{array}$} \\
\hline \multirow[b]{2}{*}{ Task } & \multicolumn{2}{|c|}{$0^{\circ}$} & \multicolumn{2}{|c|}{$\begin{array}{l}\text { Inverted } \\
\text { Flip }\end{array}$} & \multicolumn{2}{|c|}{$\begin{array}{l}\text { Inverted } \\
\text { Spin }\end{array}$} \\
\hline & $\mathrm{RT} \pm S E$ & MPE & $\mathrm{RT} \pm S E$ & MPE & $\mathrm{RT} \pm S E$ & MPE \\
\hline formal & $1,074 \pm 35$ & 2.5 & $1,248 \pm 49$ & 6.5 & $1,488 \pm 74$ & 7.5 \\
\hline Mirror image & $1,223 \pm 48$ & 8.2 & $1,277 \pm 52$ & 7.1 & $1,563 \pm 62$ & 9.2 \\
\hline$M$ & $1,148 \pm 39$ & 5.4 & $1,263 \pm 49$ & 6.7 & $1,526 \pm 65$ & 8.3 \\
\hline
\end{tabular}

puter to the nearest millisecond. The trial concluded with the experimenter entering the response into the computer as correct, incorrect, or spoiled. The naming task was preceded by eight practice trials to acquaint the subject with the procedures, as well as the use of the voice-activated relay.

Following the naming task, subjects were trained and tested on normal and mirror-image versions of the six upright objects, as in Experiment 1. Five subjects were unable to meet the criteria after several attempts and were replaced. Instruction on picture-plane and depth-plane rotation, as in Experiment 1, was next given. This training was followed by preliminary testing of mirror-normal judgments for all orientations prior to the experimental trials. As in Experiment 1, "flip" or "spin" cued the appropriate transformation for inverted objects. At all other orientations, a neutral " $x x x x "$ preceded the presentation of the objects, and subjects were expected to rotate to the upright in the picture plane. One normal and one mirror-image trial was presented at each inverted-spin, inverted-flip, and noninverted orientation for a total of 14 trials. If more than one error was made, the 14 trials were repeated. Subjects then proceeded to the experimental mirror-normal trials.

The experimental trials were presented in a single block of 84 trials. Each object was presented equally often at each invertedspin, inverted-flip, and noninverted orientation. Half the trials at each orientation required normal and half mirror-image responses. At the conclusion of the trials, subjects were asked if they felt they had used any procedure other than picture-plane and depth-plane rotation to make the required decision on spin and flip trials.

\section{Results and Discussion}

Two subjects reported using a nonrotation strategy to perform the normal-mirror judgments for trials requiring an out-of-plane flip. Two other subjects performed at chance level on inverted trials. These 4 subjects were replaced.

In the naming task, RTs of less than 300 and greater than 4,000 msec were considered outliers. Less than $1 \%$ of the naming data were rejected on this basis. Mean naming times for correct responses were calculated for each subject at each orientation. Response latencies of less than 300 and greater than $5,000 \mathrm{msec}$ in the normalmirror task were considered outliers. Application of this criterion resulted in the rejection of less than $1 \%$ of the data. Mean RTs for correct responses were calculated for each subject in each orientation and version condition.

The results from the normal-mirror task for upright, inverted-flip, and inverted-spin were first compared. This analysis provided a direct comparison with the results of Experiment 1. As can be seen in Table 2, the effect of transformation was significant. Responses to spin drawings were $378 \pm 55 \mathrm{msec}$ slower than responses to upright drawings. Similarly, responses to flip drawings were $115 \pm 35 \mathrm{msec}$ slower than upright drawings. The $263 \pm 49$-msec advantage of flip over spin was also significant, replicating the results of Experiment 1.

Responses to normal views were faster and more accurate than responses to mirror-image views, and the difference between normal and mirror-image was much greater in the upright condition $(149 \pm 31 \mathrm{msec})$ than in either the spin $(74 \pm 45 \mathrm{msec})$ or the flip conditions $(29 \pm 33 \mathrm{msec})$. This pattern of differences between normal and mirrorimage versions across conditions is in contrast to that observed in Experiment 1. The effect does not appear to be reliable and is not discussed further.

Naming versus normal mirror. The argument that individual differences exist in the rotation route adopted to identify inverted objects leads to the prediction that the distribution of the magnitude of the effect of orientation on naming for $120^{\circ}$ and $180^{\circ}$ orientations should be bimodal. If some individuals rotate inverted objects in the picture plane to produce slower RTs at $180^{\circ}$, there should be one mode centered above zero in the distribution of scores. Similarly, if other individuals rotate inverted objects in the depth plane to produce faster RTs at $180^{\circ}$, there should be a second mode centered below zero in the distribution of scores. As can be seen in Figure 2 , the distribution of orientation effect scores does suggest two distributions, with one mode above zero and one below, as would be predicted.

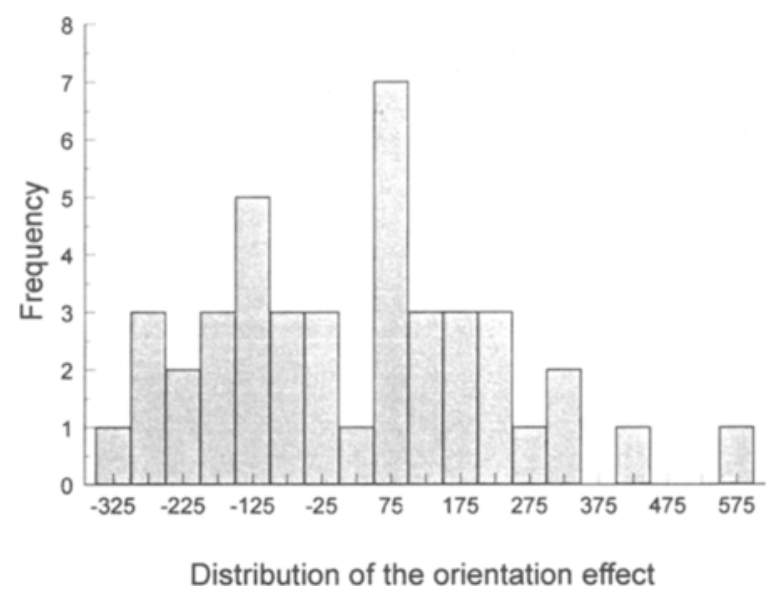

Figure 2. Distribution of the magnitude of the orientation effect on naming time for $120^{\circ}$ (mean of clockwise and counterclockwise rotations) and $180^{\circ}$ orientations in Experiment 2 . The histogram is based on a bin size of 50 msec. 
On the basis of these RT data for naming trials, two groups of subjects were defined. Subjects whose mean naming times at $180^{\circ}$ were greater than the average of naming times at $120^{\circ}$ and $240^{\circ}$ were placed into one group and referred to as spinners. Twenty-two subjects fell into this category. Subjects whose mean naming times at $180^{\circ}$ were less than the average of naming times at $120^{\circ}$ and $240^{\circ}$ were placed in a second group and referred to as flippers. There were 20 subjects of this type.

Performance on the naming and normal-mirror tasks was compared across the six orientations $\left(0^{\circ}, 60^{\circ}, 120^{\circ}\right.$, $180^{\circ}, 240^{\circ}$, and $300^{\circ}$ ) for spinners and flippers. The results for spinners are shown in Figure 3. (Note that the results for $0^{\circ}$ and $360^{\circ}$ represent the same data and are presented to help judge the symmetry around $180^{\circ}$. The data for $0^{\circ}$ were considered only once in any analysis.) The normalmirror function was plotted using inverted-spin data for the $180^{\circ}$ orientation. Figure 3 also shows the mean invertedflip RT plotted as a separate point. As is evident, naming time was faster than the time to make the normal-mirror judgment, and RT increased with increasing departure from the upright. The component of the orientation effect reflecting the linear effect of orientation was assessed by applying the contrasts $-3,-1,1,3,1$, and -1 to the results for the six orientations. RT increased linearly as objects were rotated farther from the upright $\left[F(1,21)=51.17, M S_{\mathrm{e}}=52,762, p<.0001\right]$. This fit accounted for $94.5 \%$ of the variance. The residual variance in response latencies after the variance for the linear trend was removed was not significant $\left[F(4,84)=1.14, M S_{\mathrm{e}}=\right.$ $34,329, p>.10]$. There was no difference in the orientation effect across the two tasks. Similarly, the slopes of the linear effect of orientation did not differ across the

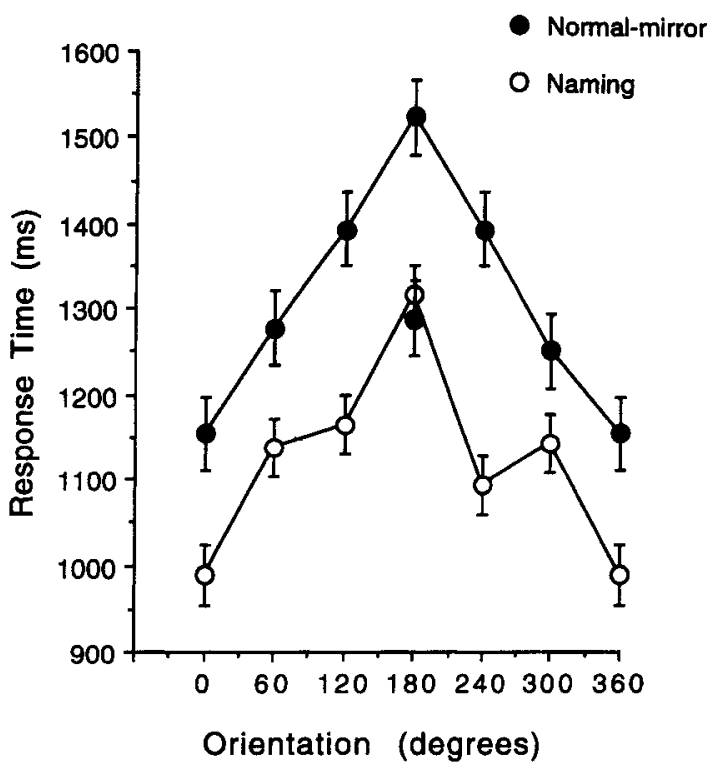

Figure 3. Mean response time at each orientation for spinners in the naming task (open circles) and in the normal-mirror task (filled circles) in Experiment 2. The isolated point represents the mean RT for inverted objects under flip instructions. Error bars are within-subjects error (Loftus \& Masson, 1994) calculated separately for each function.

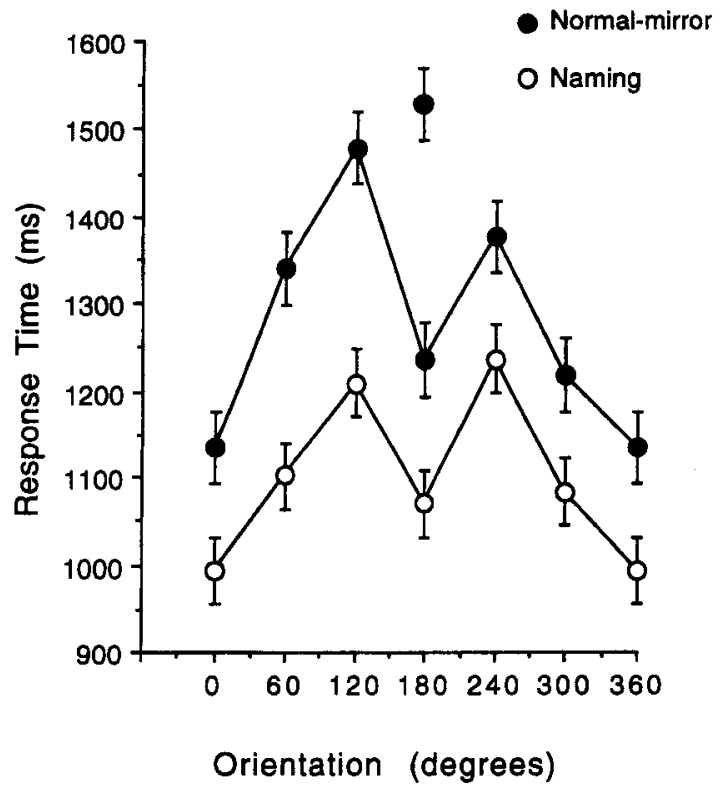

Figure 4. Mean response time at each orientation for flippers in the naming task (open circles) and in the normal-mirror task (filled circles) in Experiment 2. The isolated point represents the mean RT for inverted objects under spin instructions. Error bars are within-subjects error (Loftus \& Masson, 1994) calculated separately for each function.

two tasks as assessed by the task by orientation $n_{\text {linear }}$ interaction $\left[F(1,21)=2.38, M S_{\mathrm{e}}=35,331, p>.10\right]$. The least squares estimate of rate of rotation for the normalmirror task was $485^{\circ} / \mathrm{sec}$ (slope, $2.06 \pm .39 \mathrm{msec} / \mathrm{deg}$ ). The corresponding rate for the naming task was $617 \% \mathrm{sec}$ (slope, $1.62 \pm .27 \mathrm{msec} / \mathrm{deg}$ ).

The data for flippers are shown in Figure 4. The normalmirror function was plotted with the inverted-flip data for the $180^{\circ}$ orientation, with the mean RT for inverted-spin plotted as a separate point. As was the case for spinners, RTs were faster in the naming condition and increased as a function of orientation. The linear component of the orientation effect was significant $\left[F(1,19)=30.84, M S_{\mathrm{e}}=17,470\right.$, $p<.0001]$, accounting for $24.2 \%$ of the variance. The RTs at $180^{\circ}$ were faster than would be predicted on the basis of a linear increase in RTs, and this deviation from linearity was significant $\left[F(4,76)=11.69, M S_{\mathrm{e}}=36,002, p<\right.$ $.0001]$. As is evident in Figure 4, the effect of orientation did not differ for the naming and normal-mirror tasks, nor was there any difference in the linear component $(F<1)$. The least squares estimate of rate of rotation for the normal-mirror task was $1,316^{\circ} / \mathrm{sec}$ (slope, . $76 \pm .24 \mathrm{msec} / \mathrm{deg}$ ). The corresponding rate for the naming task was $1,667 \% \mathrm{sec}$ (slope, $.60 \pm .16 \mathrm{msec} / \mathrm{deg}$ ). When the data for $180^{\circ}$ were excluded and estimates were based on the remaining orientations of $0^{\circ}$ to $120^{\circ}$, the rates were for normal-mirror and naming were $410^{\circ} / \mathrm{sec}$ (slope, $2.44 \pm .41 \mathrm{msec} / \mathrm{deg}$ ) and $521^{\circ} / \mathrm{sec}$ (slope, $1.92 \pm .29 \mathrm{msec} / \mathrm{deg}$ ), respectively.

Error rates for spinners and flippers for the two tasks are given in Table 3. Accuracy was higher in the naming task than in the normal-mirror task for both spinners and 
Table 3

Mean Percent Error for Each Naming Group (Spinners/Flippers) at Each Orientation and in Each Task (Naming/Normal-Mirror) of Experiment 2

\begin{tabular}{|c|c|c|c|c|c|c|}
\hline \multirow[b]{2}{*}{ Task } & \multirow[b]{2}{*}{$0^{\circ}$} & \multicolumn{5}{|c|}{ Orientation } \\
\hline & & $60^{\circ}$ & $120^{\circ}$ & $180^{\circ}$ & $240^{\circ}$ & $300^{\circ}$ \\
\hline \multicolumn{7}{|c|}{ Spinners } \\
\hline Naming & 0.8 & 3.5 & 1.7 & 0.8 & 1.5 & 4.0 \\
\hline Normal mirror & 5.8 & 5.0 & 6.8 & $7.0 / 9.1$ & 8.5 & 3.5 \\
\hline \multicolumn{7}{|c|}{ Flippers } \\
\hline Naming & 1.7 & 0.0 & 2.5 & 5.1 & 2.5 & 0.8 \\
\hline Normal mirror & 4.8 & 4.9 & 10.5 & $6.3 / 5.9$ & 5.9 & 7.5 \\
\hline
\end{tabular}

Note-Error rates for the normal-mirror task under flip and spin instructions are provided separately.

Table 4

Mean Response Time Difference (in Milliseconds) Between $0^{\circ}$ and $180^{\circ} \pm$ Standard Error for Each Group in the Naming Task and the Flip and Spin Conditions of the Normal-Mirror Task of Experiment 2

\begin{tabular}{lccc}
\hline Group & Name & Flip & Spin \\
\hline Spinners & $326 \pm \mathbf{4 6}$ & $124 \pm 77$ & $369 \pm 48$ \\
Flippers & $76 \pm 31$ & $100 \pm 82$ & $394 \pm 52$ \\
\hline
\end{tabular}

flippers. The only significant effect of orientation was in the normal-mirror task for the spinners, where accuracy decreased with increases in orientation. There was no evidence of any speed--accuracy tradeoffs.

To directly compare performance on naming, flipping, and spinning, differences between mean RTs for $180^{\circ}$ and $0^{\circ}$ were calculated for each subject in the flipper and spinner groups. Three difference scores were obtained for each subject; one for naming trials, one for flip trials, and one for spin trials in the normal-mirror task. Mean differences for the two groups are given in Table 4 . As is evident, there was a significant difference between name and flip but no difference between name and spin in the spinner group. For flippers, the pattern of effects was different, as expected; name and spin differed from each other, but name and flip did not.

\section{GENERAL DISCUSSION}

The present studies yield several important results. First, images of inverted natural objects can be rotated to the upright in either the picture plane or the depth plane for comparison with stored canonical representations in order to make normal-mirror judgments. Second, the process of rotation in the depth plane is much faster than that in the picture plane. Third, subjects who showed relatively fast naming times at $180^{\circ}$ exhibited overall identification functions that are similar in shape and slope to those found for the normal-mirror task under instructions to mentally flip inverted objects. Fourth, subjects who showed relatively slow naming times at $180^{\circ}$ exhibited overall identification functions that do not differ in overall shape and slope from the normal-mirror function where inverted objects were mentally rotated to the upright.
Although the degree of rotation required to transform a familiar object to the upright is identical under the two types of rotation instruction, the apparent rate of rotation is much more rapid for depth rotation than for pictureplane rotation. This finding for a task requiring normalmirror discriminations is consistent with past work. Similar effects have been found previously for unfamiliar, Shepard and Metzler 3-dimensional cube figures (Metzler \& Shepard, 1974; Parsons, 1987). The present results demonstrate that these transformation processes can also be used to make decisions about objects that have wellestablished internal representations.

The results also provide an explanation for an earlier finding. Murray (1995a) asked subjects to imagine an upright object at another cued orientation, a task generally considered to require mental rotation. Murray found that image formation time increased in a predictable linear fashion for orientations from $0^{\circ}$ to $120^{\circ}$. However, at $180^{\circ}$, image formation times were much faster than at orientations $120^{\circ}$ from upright, with 20 of the 24 subjects showing such an effect. In the task, the subject was cued as to the required orientation by an arrow that followed the display of the upright object. For inverted objects, this cue took the form of a downward pointing arrow. It is possible that the arrow served not only as a cue to the required orientation for the imagined object, but also as a cue to rotation route. The appearance of a downward pointing arrow would be highly suggestive of a flip from the upright to the inverted position and may have provided sufficient inducement to rotate in the depth plane.

The clear implication of the finding of faster RTs following instructions to rotate in depth is that the processes involved in rotation about the horizontal axis are easier to enact and/or are faster. Why this might be the case is not clear. One possibility is that the left and right handedness of the parts of an object relative to the observer do not alter in a rotation in depth (Parsons, 1987). This consistency throughout the path of rotation may allow for easier maintenance of the spatial relations among features of the rotating object image for comparison with stored representations, and result in a more efficient rotation process.

Most importantly, the results suggest that the two different rotation routes to the upright from $180^{\circ}$ are also used to identify objects in a task where subjects are free to use any processing strategy. One group of subjects seems to have adopted a strategy of rotation through the picture plane, and the other group seems to have used rotation through the depth plane. The close correspondence between the magnitude of the orientation effects at $180^{\circ}$ in the naming task and the normal-mirror task under spin instructions for relatively slow identifiers, and a similar correspondence for relatively fast identifiers under flip instructions, is entirely consistent with this interpretation.

An alternative explanation of faster naming times for objects at $180^{\circ}$ involves nontransformation processes that extract orientation-invariant features for identification of inverted objects (Jolicoeur, 1990). However, it is not 
clear why identification through extraction of orientationinvariant features would produce the pattern of effects exhibited by the present two groups of subjects. The bimodal distribution of the magnitude of the orientation effect on naming time for $\pm 120^{\circ}$ and $180^{\circ}$ is not readily explained under an orientation-invariant features explanation. Additionally, the similarity of the pattern of orientation effects for spinners and flippers in comparison with spinning and flipping performance in the normalmirror task must also find an explanation in an orientationinvariant features account.

One possibility is to consider that normal-mirror decisions for $180^{\circ}$ objects were arrived at without either spinning or flipping transformations. Perhaps subjects learned rules such as, "In the flip condition, look for the critical feature for this object on the same side as the normal upright; in the spin condition, look for the feature on the opposite side." Although this is a possibility, other considerations suggest that subjects did not adopt this nontransformation strategy. First, the stimuli were carefully chosen and paired with respect to overall visual similarity to minimize the ease with which a nontransformation strategy could be developed and used. For example, the kettle and watering can both have spouts, but on the opposite sides. Similarly, the cup and frying pan both have handles, again on opposite sides. The third pair, spinning wheel and harp, are both complex and perhaps asymmetrically defined more on the basis of global shape than on the basis of any particular feature. Second, all subjects were queried at the conclusion of the session as to how they had performed the task, and only 2 of the subjects reported developing nontransformation strategies, with all other subjects indicating that they had imagined the objects rotated to the upright in the cued plane. Third, spin instructions resulted in the expected mental rotation function, suggesting that subjects were rotating inverted objects in the picture plane, as instructed. The functions peaked at $180^{\circ}$ and the magnitudes of the orientation effect (369-msec difference between $0^{\circ}$ and $180^{\circ}$ for spinners, 396-msec difference for flippers) are comparable to those obtained in earlier mental rotation studies (Shepard \& Cooper, 1982). Finally, the pattern of faster decision times for inverted objects under flip instructions is entirely consistent with previous studies where subjects mentally rotated 3-dimensional cube figures (Metzler \& Shepard, 1974; Parsons, 1987). Additionally, the M-shaped function found for flippers is comparable to that observed in the image formation task described above (Murray, 1995a) in which mental rotation is required to form the nonupright images. In sum, the evidence suggests that subjects did perform the normal-mirror task by rotating the drawings through the picture and depth planes.

Why subjects in the naming task do not uniformly choose the faster rotation route in the depth plane is not evident. One possibility is suggested by the work of Just and Carpenter (1985), who found that individuals of high spatial ability imagine more efficient spatial transformations than do individuals of low spatial ability. Parsons
(1987) failed to replicate this result in his experiment but noted that the restricted range of moderate-to-high spatial ability in his sample was possibly not broad enough to detect the expected differences in transformation abilities. An exploration of general spatial abilities in the context of the present findings may well yield some informative results. It should be noted that in the present studies, flippers and spinners did not differ in their abilities to perform rotations in the depth and picture planes when specifically instructed to perform the two types of rotation.

The present results provide further evidence that spatial transformation procedures are used to align input representations with orientation-specific representations in memory. Consistent with previous findings (Jolicoeur, $1985,1988)$, the results for orientations from $0^{\circ}$ to $120^{\circ}$ of clockwise or counterclockwise rotation show similar slopes of the orientation effect on RTs across a naming task and a task requiring a normal-mirror judgment. This similarity between the two functions suggests that a common mechanism, namely mental rotation, underlies the orientation effect in the two tasks. Some decision about either the object's identity or its view along a left-right dimension requires a spatial transformation of an image of the rotated object to the upright for comparison with a canonical representation. This transformation appears to take the form of a rotation in the picture plane. For both decisions, the transformation is carried out in order to provide an internal representation that encodes the spatial relations among object features and parts. The results for objects at $180^{\circ}$ suggest that naming and left-right discriminations are also accomplished by transformation of an object image to the upright for comparison with orientation-specific representations. However, in the unconstrained task of identification, the form of the transformation can be either a rotation in the picture plane or a faster rotation in the depth plane about the horizontal axis of the inverted object. Selection of the particular transformation route used appears to be subject to individual differences.

\section{REFERENCES}

Bressan, P., \& Vicario, G. B. (1984). On misoriented letters. Perception, 13, 687-694.

Jolicoeur, P. (1985). The time to name disoriented natural objects. Memory \& Cognition, 13, 289-303.

JoLICOEUR, P. (1988). Mental rotation and the identification of disoriented objects. Canadian Journal of Psychology, 42, 461-478.

JOLICOEUR, P. (1990). Identification of disoriented objects: A dualsystems theory. Mind \& Language, 5, 387-410.

JolicoEUR, P., \& MiLLIKEN, B. (1989). Identification of disoriented objects: Effects of context of prior presentation. Journal of Experimental Psychology: Learning, Memory, \& Cognition, 15, 200-210.

Just, M. A., \& Carpenter, P. A. (1985). Cognitive coordinate systems: Accounts of mental rotation and individual differences in spatial abilities. Psychological Review, 92, 137-172.

Koriat, A., Norman, J., \& Kimchi, R. (1991). Recognition of rotated letters: Extracting invariance across successive and simultaneous stimuli. Journal of Experimental Psychology: Human Perception \& Performance, 17, 444-457.

Loftus, G. R., \& Masson, M. E. J. (1994). Using confidence intervals in within-subject designs. Psychonomic Bulletin \& Review, 1, 476-490. 
MAKI, R. H. (1986). Naming and location of tops of rotated pictures. Canadian Journal of Psychology, 40, 368-387.

Metzler, J., \& ShePard, R. N. (1974). Transformational studies of the internal representation of 3-dimensional objects. In R. L. Solso (Ed.), Theories of cognitive psychology: The Loyola Symposium (pp. 147-201). Potomac, MD: Erlbaum.

MURRAY, J. E. (1995a). Imagining and naming rotated natural objects. Psychonomic Bulletin \& Review, 2, 239-243.

Murray, J. E. (1995b). The role of attention in the shift from orientation-dependent to orientation-invariant identification of disoriented objects. Memory \& Cognition, 23, 49-58.

Murray, J. E., Jolicoeur, P., McMullen, P. A., \& Ingleton, M. (1993). Orientation-invariant transfer of training in the identification of rotated natural objects. Memory \& Cognition, 21, 604-610.

Parsons, L. M. (1987). Visual discrimination of abstract mirrorreflected 3-dimensional objects at many orientations. Perception \& Psychophysics, 42, 49-59.

SCHNEIDER, W. (1988). Micro Experimental Laboratory: An integrated system for IBM PC compatibles. Behavior Research Methods, Instruments, \& Computers, 20, 206-217.

SHEPARD, R. N. (1975). Form, formation and transformation of internal representations. In R. L. Solso (Ed.), Information processing and cognition: The Loyola Symposium (pp. 87-117). Hillsdale, NJ: Erlbaum

SHEPARD, R. N., \& COOPER, L. A. (1982). Mental images and their transformations. Cambridge, MA: MIT Press.

Shepard, R. N., \& METZler, J. (1971). Mental rotation of 3-dimensional objects. Science, 171, 701-703.

SNODGRAss, J. G., \& VANDERWART, M. (1980). A standardized set of 260 pictures: Norms for name agreement, image agreement, familiarity, and visual complexity. Journal of Experimental Psychology: Human Learning \& Memory, 6, 174-215.
TARr, M. J., \& Pinker, S. (1989). Mental rotation and orientationdependence in shape recognition. Cognitive Psychology, 32, 193-254.

Ullman, S. (1989). Aligning pictorial descriptions: An approach to object recognition. Cognition, 32, 193-254.

\section{NOTE}

1. All results reported throughout the paper were confirmed in analyses of variance; these results are available on request.

\section{APPENDIX}

Names of objects used in the naming task in Experiment 2. Actual line drawings can be seen in Snodgrass and Vanderwart (1980).

$\begin{array}{llll}\text { Airplane } & \text { Cow } & \text { Harp } & \text { Rhinoceros } \\ \text { Ashtray } & \text { Cup } & \text { Horse } & \text { Sailboat } \\ \text { Bed } & \text { Deer } & \text { Kettle } & \text { Seal } \\ \text { Bird } & \text { Dog } & \text { Lion } & \text { Sheep } \\ \text { Blouse } & \text { Donkey } & \text { Mouse } & \text { Spinning Wheel } \\ \text { Camel } & \text { Duck } & \text { Nose } & \text { Swan } \\ \text { Chair } & \text { Foot } & \text { Penguin } & \text { Toaster } \\ \text { Church } & \text { Frying Pan } & \text { Pig } & \text { Wagon } \\ \text { Coat } & \text { Goat } & \text { Rabbit } & \text { Watering Can }\end{array}$

(Manuscript received May 31, 1995 ; revision accepted for publication November 5, 1995.) 